FORMATION Formation emploi

Revue française de sciences sociales

130 | Avril-Juin 2015

L'essor des compétences non académiques

\title{
Les compétences non académiques en question
}

Soft skills in debate

Fragen zu den nichtakademischen Kompetenzen

Las competencias no académicas en cuestión

Marie Duru-Bellat

\section{OpenEdition}

Journals

Édition électronique

URL : http://journals.openedition.org/formationemploi/4393

DOI : 10.4000/formationemploi.4393

ISSN : 2107-0946

Éditeur

La Documentation française

Édition imprimée

Date de publication : 10 juin 2015

Pagination : 13-29

ISSN : 0759-6340

Référence électronique

Marie Duru-Bellat, «Les compétences non académiques en question», Formation emploi [En ligne],

130 | Avril-Juin 2015, mis en ligne le 08 juin 2017, consulté le 30 octobre 2020. URL : http://

journals.openedition.org/formationemploi/4393; DOI : https://doi.org/10.4000/formationemploi.4393

(C) Tous droits réservés 


\title{
Les compétences non académiques en question
}

\author{
Marie Duru-Bellat \\ Sociologue spécialiste des questions d'éducation et de stratification sociale, \\ professeur émérite à Sciences-Po (Paris) et chercheur à l'Observatoire sociologique du \\ changement et à l'Institut de recherche en éducation (Dijon)
}

Résumé

\section{Les compétences non académiques en question}

Le texte discute la notion de compétences «non académiques ». Cette notion ne constitue pas une rupture dans les questionnements sociologiques et pédagogiques; en outre, malgré les débats vifs qu'elle suscite, elle apparaît aujourd'hui extrêmement mobilisée dans les analyses et les pratiques du marché du travail. Même si l'évaluation des compétences non académiques s'avère difficile, même si cette notion reste problématique d'un point de vue théorique, sa prise en compte par les acteurs de la formation apparaît incontournable.

Mots clés : Compétence, sociologie de l'éducation, socialisation, savoir-être, contenu de formation

Abstract

\section{Soft skills in debate}

The paper questions the notion of « soft skills». This notion does not bring in a breach in the sociological and pedagogical analysis; besides, whatever the lively disputes it generates, it is today very currently used in the analysis and practices of the labor market. Even if assessing these soft skills proves especially difficult, even if this notion remains theoretically problematic, one may consider that none of the actors involved in education and training may discard it.

Keywords: skill, educational sociology, socialisation, know-how-to-be, training content Journal of Economic Literature: I 21, J 24, M 59

Traduction : Auteure 
La notion de compétences est aujourd'hui très répandue dans le monde de la formation et du travail ${ }^{1}$, quand il s'agit de comprendre pourquoi et comment tel individu se montre capable d'une performance donnée. On peut cependant se demander s'il s'agit là d'une rhétorique vraiment nouvelle - faisant écho à des transformations socio-économiques contemporaines -, ou si elle ne fait que remettre sur le devant de la scène des interrogations classiques sur les systèmes éducatifs et les questions de formation.

Cette interrogation vaut particulièrement pour la notion de compétences " non académiques " ou transversales (telles que la résolution de problème, la maîtrise des communications sous toutes leurs formes, la capacité à vivre ensemble...). On désigne ainsi des compétences qui se développent d'une part, au travers de l'ensemble des activités de formation (initiale et continue) - en incluant les enseignements disciplinaires mais sans s'y limiter - et d'autre part, du fait des expériences diverses de la vie, professionnelle et personnelle. Elles s'opposent ainsi aux compétences issues des savoirs académiques ou techniques, eux-mêmes inscrits dans des corpus scientifiques et diffusés dans un cadre formel.

Mais on peut tout autant questionner la notion de compétences académiques, ces compétences censées découler des savoirs disciplinaires acquis - on saura calculer ses chances de gagner au loto parce que l'on maîtrise le calcul des probabilités -, auxquelles les compétences non académiques sont volontiers opposées.

S’il s'avère, ce que nous montrerons dans une première partie, que la notion de compétences non académiques s'inscrit dans la lignée de la vocation socialisatrice que s'est toujours donnée l'éducation, il convient d'analyser, comme nous le ferons en deuxième partie, pourquoi elle suscite autant de réactions virulentes.

Peut-être faut-il, et nous suivrons cette piste dans une troisième partie, au-delà des raisons idéologiques, invoquer des transformations objectives du monde du travail pour comprendre la montée irrépressible des compétences, particulièrement des compétences non académiques. En conclusion, nous soulignerons que même si la notion de compétences reste problématique d'un point de vue intellectuel - ce qui complexifie la tâche des chercheurs qui tenteraient d'évaluer lesdites compétences - cela ne dispense pas de les intégrer aujourd'hui dans une réflexion plus générale sur les contenus de formation.

1. Voir la bibliographie du numéro de la revue Formation Emploi consacré aux « Usages sociaux de la compétence $"\left(n^{\circ} 99,2007\right)$. 


\section{Développer des compétences, une rhétorique (vraiment) nouvelle au cœur des questions éducatives?}

Léducation a toujours eu, aux yeux des sociologues, une vocation " non académique ", évidente pour les philosophes (voir par exemple Kambouchner, 2013) et plus encore pour les pédagogues. Mais sa " découverte " par les organisations internationales, notamment l'OCDE (Organisation de coopération et de développement économiques), est plus récente.

\section{1 Éduquer ou transmettre un halo de compétences « non académiques "...}

Aux yeux des premiers sociologues de l'éducation comme Durkheim, l'école, en tant qu'institution, a pour fonction essentielle d'inculquer aux enfants les valeurs morales qui constituent le ciment de la société. La socialisation scolaire est avant tout une éducation morale et culturelle, même si, en sus de cette fonction intégratrice, l'école doit également, fonction plus technique, préparer chacun à une place dans la division sociale du travail.

Cette visée socialisatrice s'exprime par maints détails de l'organisation scolaire. Ainsi, comme l'expriment explicitement les programmes des écoles primaires de 1832, arriver à l'heure, renoncer au jeu pour entrer en classe, observer le silence, tout cela oblige l'élève à une série de petits triomphes sur ses penchants ». On n'est pas très loin de la capacité à " gérer le stress ", volontiers considérée comme une compétence non académique !

De l'autre côté de l'Atlantique, le sociologue Parsons souligne lui aussi la visée socialisatrice de l'école : extrayant l'enfant de l'univers familial, elle lui fait intérioriser ce qui est considéré comme beau, bien, légitime dans la société dont il est membre. L'école a alors, de ce fait, une fonction clairement idéologique. Aux États-Unis, l'individu y apprend notamment à valoriser la logique de l'accomplissement individuel, et à considérer comme évident que la sélection scolaire et sociale repose sur des critères de compétence ; chacun obtient alors le statut qu'il mérite, et cette idéologie méritocratique permet de légitimer les hiérarchies sociales.

Ces valeurs que l'école transmet, et plus largement les fondements de la socialisation qui y prend place, vont être, à partir des années 1960, au cœur des analyses de sociologues comme Bourdieu et Passeron. À leurs yeux, le rôle effectif de l'école n'est pas de forger un consensus reposant sur des valeurs partagées ; de fait, les groupes dominants la modèlent et l'utilisent pour reproduire leur position, conformément à leurs intérêts particuliers. Pour ce faire, ces groupes diffusent des valeurs et des schèmes de pensée, donc du "nonacadémique ", qui vont informer tout un "curriculum caché ", soit tout ce qui va être inculqué au-delà de l' " académique " explicitement au programme. Ainsi, à travers des automatismes de raisonnement ou de grilles pour résoudre les problèmes, l'école dote les élèves d'un mode de fonctionnement intellectuel permettant la communication sur la 
base d'un "sens commun » constituant l' "habitus cultivé » - en d'autres termes, la manière, largement inconsciente, de penser - d'une époque (Bourdieu, 1967).

Au-delà de ces grilles intellectuelles communes, les groupes qui contrôlent l'école vont aussi sélectionner les compétences qui seront au cœur de la réussite, de telle sorte que seuls les « héritiers " réussissent à les maitriser, et ce à des fins de reproduction sociale. Il peut certes s'agir de compétences académiques (maitriser la culture littéraire classique par exemple) ; cependant, les compétences non académiques sont en la matière cruciales, qu’il s'agisse d'adopter un certain rapport (distancié) au langage, de fait familier des « dominants" (Lahire, 1993), ou de développer certaines attitudes. C'est ainsi que les sociologues américains Bowles et Gintis (1977) soutiennent que l'école vise à modeler la personnalité des élèves, auxquels elle inculque des "savoir-être " adaptés à leur rôle social anticipé : aux futurs ouvriers, on apprend surtout la soumission à la règle, alors qu'auprès des futurs responsables, on valorise l'autonomie dans le travail et la créativité, à l'instar de ce qui est requis dans le monde du travail ; on pense aussi à ce rapport au temps dominé par le sens de l'urgence, inculqué, en France, au sein des classes préparatoires aux grandes écoles (Darmon, 2013).

En bref, la tradition et la critique sociologiques postulent depuis toujours que l'école inculque à la fois de l'académique et du non-académique, de fait parfois difficiles à démêler, et tout autant arbitraires en ce qu'ils ne relèvent d'aucune nécessité foncière.

Parmi les innombrables définitions possibles des contenus de formation, ce qui est retenu, dans un contexte donné, renvoie à la culture des groupes qui contrôlent l'école et aux exigences de la société, notamment des employeurs, avec à la clé un fonctionnement social sans heurt, voire une justification de l'ordre existant.

Si cette tradition sociologique n'utilise pas le terme de compétence, mais de valeurs, d'habitus, ou de curriculum caché, le type de questionnement qu'elle met en œuvre apparaît tout à fait pertinent quand on parle aujourd'hui de compétences, particulièrement des moins académiques d'entre elles. Il convainc de la nécessité d'analyser de manière aussi critique les dimensions non académiques et les dimensions académiques des formations, dans leur genèse, leur fonction idéologique et les exigences en termes de réussite qui en découlent pour les formés.

\subsection{Une montée récente et mondialisée de la notion de compétences}

En France, depuis les années 1980, la notion de compétence s'est installée au cœur des débats sur les questions de formation initiale, pour diverses raisons. Il s'est agi d'abord, suite à l'arrivée de la gauche au pouvoir, de reconnaitre que l'école n'a plus le monopole de la transmission des connaissances et que tous les savoirs (et la sélection qu'en font les programmes scolaires) sont éminemment discutables. La critique de l'académisme et de l'élitisme de la culture scolaire a été confortée par la diffusion des thèses de Bourdieu et Passeron, et le rapport « Bourdieu-Gros » de 1989 ("Principes pour une réflexion sur les 
contenus de l'enseignement "), défendant, à des fins de démocratisation, la primauté des apprentissages dits transversaux sur les savoirs disciplinaires. Cette perspective s'impose d'autant plus qu'avec la généralisation de l'enseignement secondaire, le lycée n'accueille plus uniquement des " héritiers ": non seulement ces nouveaux élèves sont plus éloignés de la culture scolaire classique, mais la question s'insinue de savoir s'ils ont vraiment besoin de la culture classique au programme pour s'insérer ensuite de manière satisfaisante dans leur vie d'adulte. On redécouvre les questionnements anciens de certains pédagogues progressistes comme Decroly, Dewey ou Freinet. Ils prônaient de lutter contre la fragmentation des connaissances et des apprentissages, grâce à une pédagogie par centres d'intérêt, substituant à la hiérarchie des savoirs une représentation plus horizontale de la diversité des compétences, susceptible de donner une chance à chacun. La notion de compétence semblait ainsi augurer d'une véritable avancée sociale...

Ces débats pédagogiques se nichent aussi dans un contexte de relatif dégel des relations entre l'école et les entreprises. En atteste la volonté de revaloriser les formations professionnelles, le développement de la formation continue ainsi que les diverses formes d'alternance et de collaboration avec le monde du travail. Là aussi, l'objectif affiché est de promouvoir la justice sociale : les inégalités qui marquent la réussite scolaire étant à présent largement connues, on compte sur la formation continue, et plus largement sur la réussite professionnelle, pour constituer un contrepoids bienvenu.

C'est sans doute pour cette raison que l'éclosion rapide de la notion de compétences dans le monde professionnel (surtout à partir des années 1990) y a suscité moins de débats critiques que dans le champ de la formation initiale. Toujours est-il que l'on importe dans le milieu scolaire des questionnements jusqu'alors tabous, en tout cas incongrus : est-ce sur la seule base de savoirs académiques qu'une personne peut évoluer dans sa carrière ? De quoi a-t-on besoin pour s'insérer dans la " vraie vie » ? Et si l'alternance pouvait être " éducative " ? Ces interrogations inédites vont de pair avec tout un arsenal de nouveaux concepts pédagogiques : interdisciplinarité, objectifs, contrat, partenariat, compétences transversales, visant tous à rendre plus transparents, plus évaluables et donc in fine (espèret-on) plus démocratiques les processus de formation.

Cette perspective est entérinée dans les textes : en 1992, la Chartre des Programmes ${ }^{2}$ consacre la notion de compétences exigibles en fin de formation. Concrètement, dès 1995, les nouveaux programmes de l'enseignement primaire listent les compétences attendues à l'issue de chaque cycle. Puis, avec la loi d'orientation de 2005, suivie du décret du 11 juillet 2006, est instauré le " socle commun de connaissances et de compétences ». Il énumère des compétences dont certaines sont clairement "non académiques » (mais la distinction académique/non académique n'est jamais explicite), qu'il s'agisse de l'acceptation des différences ou de l'aptitude à adapter sa communication au contexte...

2. Texte qui explicite et présente de façon accessible à un large public à la fois les objectifs de la formation, les contenus et le mode d'évaluation des enseignements primaire et secondaire. 
Cette évolution n’est pas spécifique à la France. Alors que dès 1995, l'Europe plaçait la reconnaissance des compétences au cœur de sa politique économique, et les explicite en 2000 avec la stratégie dite de Lisbonne, l'OCDE se positionne de manière encore plus affirmée sur ce terrain. En 1997, l'organisation postule qu'un certain nombre de " compétences clés " sont nécessaires à la croissance, compétences qui là encore outrepassent largement les seuls acquis académiques. On privilégie d'ailleurs les formes verbales pour souligner qu'elles se manifestent dans l'action : "se servir d'outils", "être capable d'interagir dans des groupes», ou "d'agir de manière autonome »... (OCDE, 2005).

Ceci s'inscrit dans un contexte international de mondialisation qui ouvre l'espace de comparaison et de concurrence entre les pays, y compris dans le domaine éducatif, où l'OCDE s'investit de plus en plus ouvertement. Elle pousse les pays à accroître l'efficacité de leur système éducatif pour affronter la concurrence économique. Pour cela, les pays gagnent à regarder ce que font les autres et à emprunter ce qui apparaît comme de « bonnes pratiques ", en référence aux acquis manifestés chez les élèves.

Les enquêtes PISA ${ }^{3}$, lancées en 2000 auprès des jeunes de 15 ans, reflètent cette politique, avec l'accent mis sur la notion de compétences pour la vie - ces "skills for life " dont devraient être dotés tous les jeunes au sortir de l'école obligatoire -, censées valoir pour tous les pays. L'OCDE souligne aussi l'importance de qualités telles que l'adaptabilité, la faculté de communiquer ou de faire preuve d'initiative, ceci en référence à des "besoins de l'économie " posés comme constituant un horizon indépassable. Cette rhétorique est appliquée aujourd'hui aux compétences des adultes, qui sont l'objet d'évaluations approfondies, en littératie, numératie et résolution de problèmes dans des environnements à forte composante technologique (OCDE, 2014).

\section{La critique des compétences : réticences idéologiques et questionnements pédagogiques}

D’emblée, dans le milieu scolaire, la notion de compétence - sans que la distinction académique/non académique soit explicitement au cœur des débats - a suscité des interrogations et des réticences, à la fois idéologiques et corporatistes, mais aussi pédagogiques (voir notamment Ropé et Tanguy, 1994).

Pour les enseignants, la polarisation sur la notion de compétence distille insidieusement une conception différente et des savoirs et de la vocation de l'école, ainsi que de leur

3. Program for International Student Assessment: Le Programme international de suivi des acquis des élèves évalue tous les trois ans, dans les pays de l'OCDE et un certain nombre de pays partenaires, les compétences des élèves de quinze ans; il porte non pas sur les programmes scolaires mais sur les compétences en compréhension de l'écrit, en calcul et en sciences, à partir d'exercices renvoyant à des situations de la vie quotidienne. 
propre rôle. Alors que dominait, en France, une conception de la justice consistant à favoriser l'accès de tous à un savoir doté d'une valeur intrinsèque, le changement de paradigme qui se profile prône d'individualiser l'enseignement. Chacun doit développer ses propres potentialités et sa personnalité, en acquérant des savoirs valant moins en eux-mêmes que comme ressources pour s'adapter aux exigences de sa propre vie. L'intégration à la Nation via l'accès à un savoir universel semble ainsi passer après l'adaptation aux exigences de la vie, notamment professionnelle, de chacun.

\subsection{Une obsession de l'utile}

La notion de compétence se polarise sur la question de savoir "à quoi sert " ce qu'on apprend. Cette rhétorique de l'utile est d'autant plus mobilisée qu'on estime les élèves qui restent de plus en plus longtemps à l'école, parfois malgré eux - a priori peu intéressés par la chose scolaire. De plus, le contexte difficile de l'emploi rend prioritaire, pour les familles et les élèves, le caractère « rentable » de ce qu'on apprend.

Cette obsession de l'utile est dénoncée comme susceptible de contaminer tous les savoirs scolaires, investis inégalement à cette aune. Elle est la cible virulente des défenseurs des savoirs abstraits, même si cette dénonciation de l'utile apparaît toujours quelque peu aristocratique (y-a-t-il tant de groupes professionnels qui soient à même de vivre de la seule maîtrise de savoirs abstraits ?)...

Quoi qu'il en soit, cette opposition omniprésente entre savoir inutile et savoir mobilisable dans la "vraie vie " rappelle par trop aux enseignants les propos critiques récurrents des employeurs sur l'inadaptation des formations scolaires. Ces discours suggèrent aussi, par contraste, que le monde du travail - l'entreprise au premier chef - peut avoir une valeur éducative, les enseignants se trouvant dès lors concurrencés par une masse croissante de formateurs.

Un certain réflexe corporatiste, compréhensible de la part des enseignants, imprègne donc ces critiques de la notion de compétence. Mais, sur un autre plan, si l'on peut contester le caractère démocratique des directives internationales en matière de compétences à transmettre (angle d'attaque très présent chez des auteurs tels que Kambouchner, 2013 ou del Rey, 2010), la façon dont le monde académique liste les savoirs disciplinaires à mettre au programme ne l'est pas nécessairement beaucoup plus ; en effet, les représentants des disciplines sont largement majoritaires dans les instances qui définissent les programmes, et ils sont, en la matière, juges et partie. Une véritable réflexion sur la culture à dispenser à tous exige de se poser d'autres questions, peut-être (encore) plus dérangeantes, celle du bien-fondé du monopole, en matière éducative, de l'école et des spécialistes d'une discipline, ou encore celle de la valeur formatrice d'une éducation par le " tout intellectuel ", eu égard aux exigences bien plus variées que rencontreront les jeunes dans la " vraie vie ».

Il reste que tenter de motiver les élèves par l'utile les rend... utilitaristes et risque de tuer le sens de tous les apprentissages à l'utilité incertaine. Cela débouche sur une hiérarchie des 
disciplines scolaires et des investissements afférents de la part des élèves. Cette hiérarchie est calée sur leurs cours - au sens des cours de la bourse - sur le marché du travail, dans une compétition âpre pour accéder aux filières menant aux emplois les plus attractifs.

S'y ajoutent les déceptions que la vie risque de réserver quant à la rentabilité effective de ce qui avait été présenté comme utile à un moment donné, car il est évidemment fort hasardeux de délimiter l'utile d'aujourd'hui, par rapport aux incertitudes de demain!

Surtout, quelle éducation promeut-on si l'on accepte cette dictature de l'utile ? Ainsi, la philosophe Nussbaum ${ }^{4}$ dénonce une éducation "tournée vers le profit ». Elle promeut au contraire une éducation développant l'indépendance d'esprit, l'imagination et la capacité d'empathie, invitant par exemple les élèves à analyser leurs émotions face à une œuvre d'art ou à éprouver le plaisir des découvertes gratuites...

Pour autant, l'utile ne s'oppose pas nécessairement à l'éducatif, comme en atteste la déclinaison des "compétences sociales " introduites dans le socle commun de connaissances : qu'il s'agisse du respect des règles du vivre ensemble ("participer à un débat argumenté ", par exemple) et encore de valeurs censées être partagées au sein de la République telles que le principe de laïcité, on parle de compétence mais, de fait, ne s'agit-il pas là d'un retour à Durkheim, à des valeurs dont on aimerait qu'elles soient communes ?

\subsection{De vrais questionnements pédagogiques...}

Malgré ces réticences, les compétences sont entrées à l'école, avec à la clé des difficultés concrètes : l'école ne pouvant appréhender les compétences en acte dans la "vraie vie " (notamment professionnelle), elle a inévitablement "scolarisé " les compétences qui, dans les référentiels et les livrets d'évaluation, sont devenues des étiquettes souvent très formelles. On comprend la perplexité des enseignants quand ils ont à évaluer, au vu de certaines performances, le degré de maitrise de compétences atomisées et parfois fort abstraites telles qu' " adapter sa communication en fonction du contexte »...

Le problème essentiel est de savoir ce que l'on mesure ainsi. Comme dans le cas des tests d'intelligence - à propos desquels on rappellera la célèbre boutade, de fait très sérieuse, de Binet (l'inventeur du quotient intellectuel), "l'intelligence, c'est ce que mesure mon test »-, on est, en matière de compétences, contraint à des définitions opérationnelles, dépendantes de l'instrument de mesure et des performances manifestées, jamais dépourvues d'arbitraire, cernées dans un contexte précis, situé et daté.

Mais comment être sûr que l'on appréhende véritablement une compétence consistante et stable ? Si la compétence est bien ce qui rend capable d'une performance mesurable, il y a probablement, dans nombre de situations du moins, plusieurs voies pour parvenir à un

4. Pour une présentation de ses thèses, voir www.laviedesidees.fr/L-utilite-sociale-des-humanites.html. 
résultat donné, donc plusieurs compétences ou combinaisons de compétences possibles, tant académiques que non académiques.

Ainsi, alors que les exercices de l'enquête PISA entendent appréhender des compétences en matière de "littératie ", la recherche en sciences de l'éducation montre que les performances des élèves, notamment des plus faibles sont, tout autant que par leurs compétences, affectées par des éléments de contexte tels que la forme même de l'exercice ou les thèmes mobilisés, la familiarité pouvant ainsi compenser une compétence défaillante (Bautier et al., 2006)...

Comment alors parier sur le caractère transférable d'une telle compétence ? La performance d'un élève - il parvient à comprendre tel texte écrit - garantit-elle, au-delà de l'exercice réussi, une compétence générale, valant quels que soient les textes ? Comment faire en sorte que les savoirs acquis par l'élève lui permettent ensuite de résoudre les problèmes variés qu'il rencontre ? Or, c'est cette transférabilité qui est visée : à travers les multiples épreuves d'évaluation, l’objectif pédagogique est bien la détection de compétences transférables...

En filigrane, se lit la difficile question du passage du savoir à l'action, dont débattent les pédagogues (Crahay, 2006) : la notion de compétence peut donner l'impression qu'on résout cette difficulté en invoquant des compétences de "niveau élevé " pour combler le fossé entre connaissances scolaires et savoirs requis dans la tâche réelle.

Mais la notion de compétence ne dit rien de l'articulation avec les savoirs ou les procédures intellectuelles également mobilisés dans la résolution d'une tâche donnée.

On cherche à appréhender un potentiel qui permettrait d'augurer d'une gamme large de performances, alors que fondamentalement la compétence manifestée est indissociable d'une situation (d'un exercice scolaire, d'un essai professionnel...). Il est donc impossible d'évaluer une compétence sans contexte. De plus, dès lors que la compétence est un savoir en acte, elle intègre indissociablement les leçons de l'expérience : les oppositions académiques entre théorie et pratique, spécialisation et polyvalence se brouillent et, surtout, on ne saurait caractériser une personne par la liste décontextualisée de ses compétences, comme on le faisait sur la base de sa qualification formelle.

C'est pourtant là une tentation constante, notamment dans le monde scolaire où l'invocation des compétences est peut-être une version euphémisée de la quête des dons, des aptitudes ou du mérite des élèves, contrainte par l'existence d'évaluations et de classements qui seraient ainsi légitimés (Duru-Bellat, 2009).

De même, dans le monde du travail, certains sociologues ont pu craindre que la notion de compétence ne revienne à " naturaliser " ce qui s'avère requis pour maîtriser un poste de travail, remettant ainsi en cause la notion fondamentalement sociale de qualification, garantie par les diplômes et les conventions (Zarifian, 1997) : on pourrait juger que tel ou tel employé, pourtant doté de tous les diplômes nécessaires, n’a pas la compétence requise 
par le poste, voire n'est tout simplement pas « employable " pour des raisons éminemment personnelles. Pourtant, dès lors que, selon les situations (qu'elles soient scolaires ou professionnelles), les personnes mobilisent des compétences variées - car, in fine, l'enjeu est de se comporter comme la situation l'exige à l'instant $\mathrm{t}$ et de manière socialement acceptable - tout risque de naturalisation semble au contraire écarté. Et d'un point de vue plus théorique, on ne saurait " psychologiser " la notion de compétence, puisqu'elle découle d'un jugement, d'une reconnaissance, dans un environnement forcément social. Ce qui conduit à souligner que toute compétence a une composante non académique, ou, pour le dire autrement, que c'est la notion de compétence purement académique qui est la plus problématique, voire n'a pas même de sens : tout savoir ne vaut que si l'on s'en sert avec pertinence dans telle ou telle situation...

\section{Une montée irrépressible des compétences 3 dans le monde du travail?}

Au-delà de ces débats, on peut juger que la notion de compétence (et de compétence non académique) s'impose de fait dans le monde professionnel, vu les mutations intrinsèques du travail, et pour des raisons variées (que nous ne saurions lister de manière exhaustive), tenant à la fois aux nouvelles exigences du recrutement et à l'évolution intrinsèque de nombre d'emplois.

Une première raison renvoie à ce que l'on peut considérer comme un effet de seuil en matière de formation : ce n'est pas parce que les jeunes sont de plus en plus instruits (que leur formation est plus longue, sans préjuger de sa " qualité ») qu’ils sont pour autant proportionnellement mieux préparés à la vie professionnelle. Des données récentes (on pense à la dernière enquête PISA) montrent qu'il n'y a guère de relations entre les compétences académiques en mathématiques et les compétences non académiques en résolution de problèmes concrets (calculer une réduction sur un produit en effectuant une règle de trois) ; de même d'ailleurs, les relations sont assez lâches entre le niveau d'éducation formel d'un pays et les compétences de ses adultes (OCDE, 2014).

N'est-il pas alors opportun de réfléchir à la façon d'améliorer les compétences transmises par la formation plutôt qu'à faire " plus de la même chose ", à savoir élever le niveau de compétences académiques en dispensant (encore) plus de savoirs?

Cette perspective peut s'appliquer également à la question des débouchés professionnels. Avec l'essor spectaculaire de l'enseignement supérieur et de la recherche dans les pays émergents et la concurrence mondiale qui en découle (Brown et al., 2011), il n'est pas cer-

5. P. Brown utilise l'expression de "global auction", de bourse globale, pour exprimer le fait qu'à l'heure actuelle, tous les diplômés du monde entier sont en concurrence sur un marché globalisé unique, mais avec des « prix » différents, puisque les salaires sont très inégaux d'un pays à l'autre. 
tain que dans notre pays les emplois exigeant un niveau d'étude très élevé représenteront un jour une large portion de l'emploi total. Dans le même temps, les emplois les moins délocalisables, tels que les emplois de service à la personne (ou, autre exemple, d'employé des pompes funèbres, $c f$. Bernard, 2007) exigent des qualités de contact et de gestion de ses émotions - soit des compétences non académiques indéniables telles que l'empathie qui n'ont pas de raison de varier en proportion du niveau de formation.

En outre, avec la vive concurrence qui s'instaure pour maints emplois, entre des jeunes tous relativement diplômés, les employeurs mobilisent d'autres critères de sélection et une gamme de compétences non académiques. Une enquête du Céreq (Brefn²50, mars 2008) révèle que dans les entreprises, les recruteurs utilisent effectivement le diplôme comme un signal de compétence académique minimal s'agissant des jeunes dépourvus d'expérience professionnelle. Mais ce signal est perçu comme insuffisant ; de fait, il se conjugue avec d'autres facteurs de nature variée, allant de la possibilité de bénéficier d'aides à l'emploi à des critères de motivation, de façon de s'exprimer, de disponibilité voire des qualités personnelles très " basiques " (arriver à l'heure, être capable de travailler en équipe...), même chez les très diplômés.

Cela engendre (ou accroît) un relâchement des liens formation/emploi, étudié depuis fort longtemps par les économistes, notamment quand ils s'intéressent à ce qui explique les salaires. Parmi les déterminants les plus explorés, il y a évidemment le niveau de formation, même si l'effet spécifique d'un surcroît d'éducation reste difficile à évaluer ; les individus ayant peut-être des qualités autres (non académiques) qui font qu'à la fois ils ont pu et voulu faire des études plus longues (hier) et sont mieux appréciés (aujourd'hui), sans que ces deux événements entretiennent la moindre relation.

Mais s'agit-il d'attitudes ou de compétences ? Une recherche récente montre que le niveau de compétence de base est à même d'expliquer ce qui apparaît, au vu du diplôme, comme un déclassement ou un surclassement sur le marché du travail (Branche-Seigeot et Giret, 2014) : certains individus en apparence déclassés au vu de leur diplôme auraient en fait un niveau de compétence en littératie et/ou en numératie faible, ceci pouvant expliquer cela. De nombreuses enquêtes confirment l'importance des compétences très générales ou des attitudes des employés dans leur embauche et leur rémunération, sachant que la difficulté à évaluer précisément ces compétences et attitudes tend vraisemblablement à minorer cette importance dans les estimations chiffrées. Ainsi, Bowles, Gintis et Osborne (2001) estiment que les compétences non cognitives, qui ne s'expriment pas par les diplômes possédés (ou, quand il y en a, par des évaluations des compétences académiques), comptent pour $20 \%$ dans l'impact de l'éducation sur les revenus : en moyenne, les individus les plus diplômés seraient plus capables de coopérer dans le travail et de s'adapter aux environnements nouveaux ou à des relations sociales variées, auraient plus confiance en eux. De même, Lleras (2008) démontre que les compétences sociales (qualités relationnelles, capacité à travailler en équipe...) affecteraient davantage les salaires que les compétences cognitives. 
Certes, on peut considérer qu'il s'agit là de facteurs non cognitifs assez classiques de la productivité : l'efficacité au travail ne se réduit pas à la seule mobilisation de compétences académiques ou purement techniques. Mais il s’avère aussi que les salaires rémunèrent parfois des qualités dont la relation avec la productivité est moins évidente et/ou qui peuvent interroger sur le plan éthique. C'est ainsi qu'il existe des relations entre salaires et qualités telles que l'amour-propre ou l'agressivité, ou des traits physiques comme l'apparence; en effet, on exige souvent des candidats qu'ils aient le "physique de l'emploi » et on n'exige pas la même façon de se présenter d'une vendeuse en cosmétique que d'un employé des pompes funèbres (Bernard, 2007). Le fait que, sur le marché du travail, les salaires intègrent un ensemble large de facteurs conduit à s'interroger sur la notion de discrimination : la valorisation de plus en plus fréquente de compétences non académiques peut engendrer davantage de recrutements de prime abord entachés de discrimination. Ce serait le cas, par exemple, quand la SNCF recrute préférentiellement des jeunes de quartiers dits difficiles pour jouer le rôle de médiateurs dans les gares de banlieue (Collard, 2003) ; ou encore, quand on détecte des biais de sexe, «toutes choses - prises en compte - égales par ailleurs ». Mais dans les deux cas, on peut se demander si les différences de salaire entre les jeunes (selon leur origine) ou entre hommes et femmes ne correspondent pas pour une part à la valorisation de compétences non académiques réelles (voir les travaux en cours de Bensidoun et Trancart, 2014) : on prendrait en compte des attributs autres que ceux que sanctionnent les titres scolaires (la façon de se présenter, les qualités sociales...), mais de fait porteurs d'efficacité dans le monde professionnel. Avec à la clé une tension entre efficacité et équité - chaque fois que ces attributs relèvent de la chance ou de l'origine notamment - sauf si l'école avait le pouvoir ou prenait soin d'inculquer à tous ces compétences clairement non académiques!

En tout cas, au-delà des questions de recrutement, on peut soutenir que l'évolution des emplois valorise de plus en plus des attributs non filtrés par les diplômes, avantageant ainsi certaines catégories de la population. Globalement, pour les chercheurs les plus critiques sur la notion de compétence (voir par exemple Laval et al., 2011), il s’agit là du stade ultime de l'adaptation au nouveau capitalisme (pour reprendre les thèses de Boltanski et Chiapello, 1999) : les individus doivent pouvoir s'adapter pour faire face à des exigences variées et imprévisibles, être flexibles et polyvalents, précisément pour être " employables »!

La maîtrise de savoirs abstraits, les diplômes, les qualifications certifiées une fois pour toutes ne suffisent plus dans le monde du travail ; il faut se mobiliser, s'impliquer davantage, de manière plus intense et plus autonome. Le "nouvel esprit du capitalisme " tendrait à rendre caduque la relation (proportionnelle) prévalant jusqu'alors, entre niveau d'instruction et rémunération - qui entérinait une définition du mérite comme mérite scolaire (Duru-Bellat, 2009) - pour valoriser davantage des motivations plus personnelles.

On peut ajouter qu'il y a là aussi une volonté de rationalisation et d'efficacité : pour les responsables économiques ou les constructeurs de référentiels de formation, expliciter les objectifs et les compétences attendues répond aux nouvelles exigences sociales et écono- 
miques d'évaluation (Boltanski et Chiapello, 1999). On peut évidemment critiquer ces évolutions et dénoncer leurs effets pervers. On peut aussi se demander si la notion de compétence ne se substitue pas aujourd'hui à la notion d'aptitude, qui avait fleuri lors de la révolution industrielle, alors que se développaient les emplois spécialisés de l'industrie, parce que l'on a besoin de moins en moins d'aptitudes spécifiques pointues ; en tout cas, les spécialistes du travail montrent à l'envi combien les approches adéquationnistes d'antan - telle aptitude ou telle qualification, tel emploi - ne sont plus valables (Giret, Lopez et Rose, 2005)...

\section{Conclusion : Une notion foncièrement incertaine mais socialement utile}

Cette notion de compétence, et tout particulièrement de compétence non académique, mérite d'être discutée, d'un point de vue théorique et politique ; pour autant, cela ne la rend pas sans intérêt, au contraire même, puisqu'elle fait réfléchir !

Que l'on parle de compétence académique ou (plus encore) de compétence non académique, ces notions restent entachées de fragilités théoriques essentielles, à l'instar de toutes les définitions opérationnelles, ce qui n'enlève pas toute valeur sociale à leur évaluation.

Il n’y a qu'à penser à la notion d'intelligence, éminemment discutée, voire polémique, chez les psychologues et pour autant très mobilisée dans la pratique sociale. Rappelons ici ce que disaient Binet et Simon (1905, p.194-195), à propos de leur échelle de mesure de l'intelligence (augurant du fameux QI) : " cette échelle permet, non pas à proprement parler la mesure de l'intelligence... mais un classement..., et pour les besoins de la pratique, ce classement équivaut à une mesure ». Mais une mesure de quoi ? Appréhendées sur la base d'instruments spécifiques, ces notions qui prétendent révéler des attributs non directement observables relèvent d'une inférence toujours probabiliste. L'inférence est sans doute plus assurée en matière de compétence académique : si l'on est capable d'estimer ses chances de gagner au loto, c'est très vraisemblablement du fait de compétences en calcul de probabilité, même si pour des performances plus globales - réussir un examen, a fortiori sa scolarité - l'inférence est bien plus incertaine ; en effet, au-delà d'un seuil de compétences académiques, il faut sans doute mobiliser des compétences non académiques diverses, difficiles à spécifier...

Ce flou intrinsèque à la notion de compétence pose évidemment des problèmes au chercheur qui, dès lors que l'enjeu des compétences non académiques sur le marché du travail ne semble pas mineur, s'intéresserait à leur production au sein des systèmes éducatifs.

Les recherches butent alors sur un certain nombre de difficultés : non seulement on dispose de peu d'éléments empiriques sur les compétences non académiques (dans PISA, les mesures les plus fiables ne concernent que le goût pour le travail en groupe et celui pour la compétition), mais il s'agit de mesures déclaratives, sensibles à la formulation des questions, et sans doute entachées de biais découlant de normes de désirabilité variables 
selon les pays (par exemple, déclarer son goût pour la compétition peut être malvenu dans certains contextes culturels).

Les compétences étant fondamentalement situées, d'une part, et leur appréhension dépendantes de l'instrument de mesure, d'autre part, le chercheur ne peut s'engager qu'avec une grande précaution dans des comparaisons internationales en matière de compétences non académiques (Duru-Bellat, Mons et Savina, 2012 ; Pereyra, Kotthoff et Cowen, 2011).

La recherche des liens avec le fonctionnement des systèmes éducatifs, largement encouragée par l'OCDE, si elle est émaillée des difficultés classiques de toute évaluation des " effets " de l'éducation (Baudelot et Leclerc, 2005), va être particulièrement délicate s'agissant des compétences non académiques : identifier ce qui peut avoir un impact sur les compétences, dans ce conglomérat qui constitue l'expérience scolaire des élèves, est d'autant plus difficile qu'en la matière, c'est sans doute plus l'ensemble du climat éducatif, voire l'extra-scolaire, qui importe.

Au quotidien, les élèves peuvent, par exemple, apprendre de manière diffuse des valeurs communautaires par des situations fréquentes d'apprentissage en groupe, un climat de partage, la disqualification de la compétition, l'insistance sur les connaissances communes et la culture nationale ( $c f$. Duru-Bellat $e t$ al., 2012). Il reste que ces difficultés ne devraient pas dissuader d'explorer une question importante dès lors que l'on confie à l'institution scolaire une mission éducative, autant non académique qu’académique.

Pour les spécialistes du travail, la notion de compétence demeure également, malgré ses faiblesses intrinsèques, une notion utile. Il n'est pas sans importance de décrire ce qui est requis de fait dans tel ou tel poste, de le rendre public, tout en explicitant combien ceci est socialement situé : la compétence (académique et plus encore non académique), c’est ce qui rend l'exercice professionnel efficace aux yeux des employeurs; ce n'est donc pas avant tout (du moins dans un grand nombre d'emplois) une exigence absolue, abstraite et stable du poste, mais une appréciation sociale située.

Il convient donc de faire une lecture critique de la notion de compétence, dès lors qu'elle est toujours définie in fine par un jugement de valeur. Il faut rappeler à cet égard les analyses critiques de Naville (1945) sur la notion d'aptitude, soulignant que nombre des " aptitudes requises " sont purement contingentes à certaines situations économiques, à certains besoins ponctuels et que l'on doit donc les relativiser, prendre conscience que, dans un autre mode d'organisation du travail, elles pourraient être tout autres. Cela vaut au niveau de la société dans son ensemble : si les compétences sont ce qui permet d'être évalué positivement, elles nous renvoient une image de ce que la société valorise à l'instant t. C'est ainsi que la compétence "agir de façon autonome ", listée dans DeSeCo ${ }^{6}$

6. Il s'agit d'un programme de l'OCDE visant à définir et à sélectionner les compétences clefs dont les jeunes auront besoin dans leur vie d'adulte, ce travail de réflexion étant un préalable nécessaire à l'évaluation de ces compétences. 
(OCDE, 2005) est représentative d'une certaine culture et pourrait valoir des jugements plus mitigés ailleurs, dans les pays où le conformisme du travailleur est valorisé... Les compétences valorisées sont donc toujours nichées dans un contexte culturel et normatif.

Quant aux acteurs des systèmes de formation, ils ne peuvent écarter d'un revers de main la notion de compétence dans la conception des cursus, dès lors qu'ils jugent fondamental de sortir d'une approche auto-référencée des contenus scolaires, les fondant uniquement sur les prérequis des étapes ultérieures (Perrenoud, 2006).

Si au-delà des sensibilités idéologiques, les débats autour de la notion de compétence sont si vifs, c'est bien parce qu'ils interrogent l'ambition et les modalités d'une éducation qui ne se réduit pas à la transmission de savoirs. La notion de compétence a le mérite d'expliciter les objectifs que l'on vise et par conséquent d'ouvrir le débat sur ce que doit être le projet éducatif de l'école, pour les élèves tels qu'ils sont, ainsi que son articulation avec le monde qui les attend, notamment, mais pas seulement le monde du travail.

Dans cette perspective, il serait vain de s'arc-bouter sur la distinction compétence académique/non académique. D'une part, parce que l'on peine à catégoriser certaines compétences : comment caractériser par exemple la "capacité à utiliser le savoir/les technologies et l'information de manière interactive " (OCDE, 2005) ? Ce n'est ni du " pur académique " ni du " pur non académique "; en effet, que vaudrait un savoir quelconque qu'on ne saurait ni utiliser ni communiquer? Mais aussi parce que l'on peut modifier les curricula et que l'inclusion dans les programmes de certaines compétences de prime abord non académiques ou ignorées - savoir s'occuper d'un nourrisson par exemple - peut les «scolariser » : maîtriser la puériculture deviendra une compétence académique ! La frontière est donc fluctuante.

On ne saurait non plus rejeter la notion de compétence au nom de son caractère fondamentalement conformiste. Prendre en compte les exigences de la vie n'équivaut pas à un pur conformisme, même si les compétences incorporent toujours des aspects normatifs : considérer que les élèves ne doivent pas être uniquement familiarisés aux œuvres littéraires classiques mais aussi apprendre à s'exprimer correctement n'est pas une démission entérinant un parler relâché : l'impolitesse n'est pas une compétence, mais un parler snob, critiquable à ce titre, non plus !

On peut aussi apprendre aux élèves à critiquer, à contourner certaines exigences non académiques : celui qui n'est pas doté du « look» le plus apprécié dans une situation donnée ou des atouts plus ou moins implicites découlant de telle origine sociale peut apprendre à se présenter autrement. En tout cas, il faut apprendre à tous - à tous les élèves au retour de stages par exemple - à décrypter ce qui fait, au jour d'aujourd'hui et dans tel ou tel contexte, le "mérite professionnel ", tel que révélé par les jugements du marché du travail, et son décalage avec le mérite scolaire (Duru-Bellat, 2009). Cela fait partie de la vocation éducative et formatrice de l'école si l'on ne veut pas que les compétences requises, notamment les moins académiques d'entre elles, soient de fait le privilège des « héritiers »... 


\section{Bibliographie}

Baudelot C., Leclerc F. (2005), Les effets de l'éducation, Paris, La Documentation française, 367 p.

Bautier E., Crinon J., Rayou P., Rochex J-Y. (2006), « Performances en littératie, mode de faire et univers mobilisés par les élèves ", Revue Française de Pédagogie, n 157, pp. 85-101.

Bensidoun I., Trancart D. (2014), «Inégalités entre hommes et femmes sur le marché du travail français : quels rôles des facteurs non cognitifs ? ", Journées d'étude sur les compétences non académiques, IREDU, Dijon, 30-31 Janvier.

Bernard J. (2007), "La gestion des émotions aux pompes funèbres, une compétence reconnue?", Formation Emploi, n 99, pp. 61-74.

Binet A., Simon Th. (1905), « Méthodes nouvelles pour le diagnostic du niveau intellectuel des anormaux ", L'Année Psychologique, 11, pp. 191-244.

Boltanski L., Chiapello E. (1999), Le Nouvel esprit du capitalisme, Paris, Gallimard, 843 p.

Bourdieu P. (1967), "Systèmes d'enseignement et systèmes de pensée ", Revue internationale des sciences sociales, $\mathrm{n}^{\circ} 3$, pp. 367-409.

Bowles S., Gintis H. (1977), Schooling in Capitalist America, New York : Basic Books, $340 \mathrm{p}$.

Bowles S., Gintis H., Osborne M. (2001), “The Determinants of Earnings: A Behavioural Approach”, Journal of Economic Litterature, 39, pp. 1136-1176.

Branche-Seigeot A., Giret J.-F. (2014), « Le niveau de compétences de base peut-il expliquer le déclassement ou le surclassement sur le marché du travail ", Economies et Sociétés, $\mathrm{n}^{\circ} 36$, pp. 1439-1471.

Brown Ph., Lauder H., Aston D. (2011), The Global Auction, Oxford: Oxford University Press, $198 \mathrm{p}$.

Collard D. (2003), "La médiation : une compétence ingérable », Gérer et Comprendre, 72, pp. 15-25.

Crahay M. (2006), « Dangers, incertitudes et incomplétude de la logique de la compétence en éducation, Revue Française de Pédagogie, n 154, pp. 97-110.

Darmon M. (2013), Classes préparatoires. La fabrique d'une jeunesse dominante, Paris, La Découverte, $317 \mathrm{p}$.

Duru-Bellat M. (2009), Le mérite contre la justice, Paris, Presses de Sciences Po, 166 p. 
Duru-Bellat M., Mons N., Savina Y. (2012), « Modèles éducatifs et attitudes des jeunes : une exploration comparative internationale ", Revue Française de Sociologie, n ${ }^{\circ}$, pp. 589-622.

Giret J.-F., Lopez A., Rose J. (dir.) (2005), Des formations pour quels emplois ?, Paris, La Découverte, $384 \mathrm{p}$.

Kambouchner D. (2013), L'Ecole, question philosophique, Paris, Fayard, 354 p.

Lahire B. (1993), Culture écrite et inégalités scolaires, Lyon, PUL, 310 p.

Laval C., Vergne F., Clément P., Dreux G. (2011), La Nouvelle École capitaliste, Paris, La Découverte, $275 \mathrm{p}$.

Lleras C. (2008), "Do skills and behaviours in high school matter?”, Social Science Research, $37, \mathrm{n}^{\circ} 2$, pp. 888-902.

Moncel N. (2008), "Recrutement entreprises : les débutants sont-ils victimes d'un tri trop sélectif ?”, Céreq-Bref, 250, mars.

Naville P. (1945), Théorie de l'orientation professionnelle, Paris, Gallimard, 384 p.

OCDE (2005), La définition et la sélection des compétences clés (DeSeCo), Paris, OCDE.

OCDE (2014), Des compétences pour la vie?, Paris, OCDE (www.oece.org/fr/sites/piaacfr/evaluationdescompetencesdesadultes.htm).

Pereyra M., Kotthoff H-G., Cowen R. (eds) (2011), Changing Knowledge, Changing Tests, Changing Schools, Rotterdam: Sens Publishers, 334 p.

Perrenoud Ph. (2006), « Le socle et la statue », Cahiers pédagogiques, n 439, pp. 16-18.

Rey (de) A. (2010), A l'école des compétences. De l'éducation à la fabrique de l'élève performant, Paris, La Découverte, 268 p.

Ropé F., Tanguy L. (dir.) (1994), Savoirs et compétences. De l'usage de ces notions dans l'école et l'entreprise, Paris, L'Harmattan, 243 p.

Zarifian Ph. (1997), "La compétence, une approche sociologique ", L’Orientation Scolaire et Professionnelle, $\mathrm{n}^{\circ} 3$, pp. 429-444. 